\title{
On the Waring-Goldbach problem with small non-integer exponent
}

\author{
by \\ M. Z. Garaev (Taipei)
}

1. Introduction. I. I. Piatetski-Shapiro [6] considered the following variant of the Waring-Goldbach problem. Let $c>1$ be non-integer and denote by $H(c)$ the least $k$ such that the inequality

$$
\left|p_{1}^{c}+p_{2}^{c}+\ldots+p_{k}^{c}-N\right|<\varepsilon
$$

has a solution in prime numbers $p_{1}, p_{2}, \ldots, p_{k}$ for every $\varepsilon>0$ and $N>$ $N_{0}(c, \varepsilon)$. It is proved in $[6]$ that

$$
\limsup _{c \rightarrow \infty} \frac{H(c)}{c \log c} \leq 4 .
$$

Piatetski-Shapiro also proved that if $1<c<3 / 2$ then $H(c) \leq 5$.

In this paper we sharpen the last result and prove the following theorem:

Theorem. If $1<c \leq(\sqrt{5}+1) / 2$ then $H(c) \leq 5$.

It should be pointed out that if $c$ is near to unity then $H(c) \leq 3$. More precisely D. I. Tolev [7] showed that if $1<c<15 / 14$ then $H(c) \leq 3$. Afterwards several authors sharpened Tolev's result improving on the range for $c$ (see [1], [3], [5]). The best improvement to date is due to A. Kumchev [3]; he proved that $H(c) \leq 3$ for $1<c<61 / 55$. Note that from the result of A. Kumchev and M. B. S. Laporta [4] it follows that $H(c) \leq 4$ for $1<$ $c<6 / 5$ and for almost all $c \in(1,2)$ (in the sense of Lebesgue measure). We also refer the readers to [8].

2. Auxiliary lemmas. Set $A=\left\{n \in \mathbb{Z}: P^{c-1} / 2 \leq n \leq P^{c-1}\right\}$, $B=\{n \in \mathbb{Z}: P / 10 \leq n \leq P / 5\}$.

Lemma 1. Let $1<c \leq(\sqrt{5}+1) / 2$. Then for any real $N_{1}$ the number of solutions of the inequality

2000 Mathematics Subject Classification: 11P05, 11P32. 


$$
\left|x^{c}+y^{c}-u^{c}-v^{c}-N_{1}\right|<1, \quad x, u \in A, y, v \in B,
$$

is $O\left(P^{c} \log P\right)$.

Proof. For any given integer $k$ the number $I_{k}$ of solutions of the equation

$$
\left[x^{c}\right]+\left[y^{c}\right]=\left[u^{c}\right]+\left[v^{c}\right]+k, \quad x, u \in A, y, v \in B,
$$

satisfies

$$
I_{k}=\int_{0}^{1}\left|\sum_{x \in A} \sum_{y \in B} e^{2 \pi i \alpha\left(\left[x^{c}\right]+\left[y^{c}\right]\right)}\right|^{2} e^{-2 \pi i \alpha k} d \alpha .
$$

Hence $I_{k} \leq I_{0}$. Furthermore, for any solution $x, y, u, v$ of (1), one has

$$
\left[x^{c}\right]+\left[y^{c}\right]=\left[u^{c}\right]+\left[v^{c}\right]+N_{1}+4 \theta,
$$

where $|\theta| \leq 1$, and $N_{1}+4 \theta$ is an integer. Therefore, if we prove that the number $J$ of solutions of the equation

$$
\left[x^{c}\right]+\left[y^{c}\right]=\left[u^{c}\right]+\left[v^{c}\right], \quad x, u \in A, y, v \in B,
$$

is $O\left(P^{c} \log P\right)$ then we are done. To do that we follow our work [2].

Obviously

$$
J<P^{c}+2 \sum_{1 \leq l<P^{c-1}} J_{l},
$$

where $J_{l}$ denotes the number of solutions of the equation

$$
\left[x^{c}\right]+\left[y^{c}\right]=\left[(x+l)^{c}\right]+\left[z^{c}\right], \quad x, x+l \in A, y, z \in B .
$$

In order to estimate $J_{l}$ we fix $x=x_{0}=x_{0}(l)$ such that $J_{l}<P^{c-1} J_{l}^{\prime}$ where $J_{l}^{\prime}$ denotes the number of solutions of the equation

$$
\left[x_{0}^{c}\right]+\left[y^{c}\right]=\left[\left(x_{0}+l\right)^{c}\right]+\left[z^{c}\right], \quad y, z \in B,
$$

in variables $y, z$. It then follows that

$$
y^{c}-z^{c}<\left(x_{0}+l\right)^{c}-x_{0}^{c}+2 .
$$

Since $x_{0}+l \in A, z \in B$, we have

$$
0<(y-z) P^{c-1}<c_{1} l P^{(c-1)^{2}}, \quad \text { i.e. } \quad 0<y-z<c_{1} l P^{c^{2}-3 c+2} .
$$

Therefore

$$
J_{l}<P^{c-1} \sum_{m<c_{1} l P^{c^{2}-3 c+2}} J_{l}^{\prime}(m)
$$

where $J_{l}^{\prime}(m)$ denotes the number of solutions in $z$ of the equation

$$
\left[(z+m)^{c}\right]-\left[z^{c}\right]=a, \quad z, z+m \in B,
$$

where $a=a(m, P)$ is some fixed integer.

Suppose that $z_{0}$ is the smallest solution of (4). Then for any other solution $z$ of (4),

$$
(z+m)^{c}-z^{c}<\left(z_{0}+m\right)^{c}-z_{0}^{c}+4 .
$$


This inequality can be written as

$$
c(c-1) \int_{z_{0}}^{z} \int_{0}^{m}(\phi+\psi)^{c-2} d \phi d \psi<4 .
$$

Hence

$$
0 \leq c_{2}\left(z-z_{0}\right) m P^{c-2}<4, \quad \text { i.e. } \quad J_{l}^{\prime}(m) \leq c_{3}\left(1+P^{2-c} m^{-1}\right) .
$$

In view of (3) we obtain

$$
J_{l} \ll_{c} l P^{c^{2}-2 c+1}+P \log P
$$

and therefore in view of (2),

$$
J \ll_{c} P^{c^{2}-1}+P^{c} \log P .
$$

Since $c \leq(\sqrt{5}+1) / 2$, the result follows.

For the proof of the following three lemmas see [6].

LEMMA 2. Let $s$ be a positive integer,

$$
\delta>0, \quad \Delta>s \delta, \quad \Phi_{s}(\xi)=\frac{\sin (\Delta \xi) \sin ^{s}(\delta \xi)}{\delta^{s} \xi^{s+1}}
$$

and

$$
\phi_{s}(y)=\frac{1}{\pi} \int_{-\infty}^{\infty} \Phi_{s}(\xi) e^{i \xi y} d \xi
$$

Then

$$
\phi_{s}(y)= \begin{cases}0 & \text { if }|y| \geq \Delta+s \delta, \\ 1 & \text { if }|y| \leq \Delta-s \delta\end{cases}
$$

and also

$$
0<\phi_{s}<1 \quad \text { if } \Delta-s \delta<|y|<\Delta+s \delta .
$$

Furthermore, if $k \geq 0$ is an integer and $s \geq k+2$, then

$$
g(y)=\int_{-\infty}^{\infty}\left|\Phi_{s}(\xi) \xi^{k}\right| e^{-i \xi y} d \xi=O\left(y^{-2}\right) \quad \text { as } y \rightarrow \infty .
$$

Define

$$
S(\xi)=\sum_{P / 2<p \leq 2 P} e^{i \xi p^{c}} .
$$

Lemma 3. Let $H=e^{\sqrt{\log P}}, P^{c} / 2<N_{1}<P^{c}$. Then under the assumptions of Lemma 2 we have

$$
\frac{1}{\pi} \int_{|\xi|<H P^{-c}} \Phi_{s}(\xi) S(\xi) e^{-i \xi N_{1}} d \xi \gg \frac{P^{1-c}}{\log P} .
$$


LEMMA 4. For any fixed $m$,

$$
\max _{H P^{-c}<\xi<\log P}|S(\xi)| \ll \frac{P}{\log ^{m} P} .
$$

3. Proof of the Theorem. The proof is similar to one in [6]. Let $N>N_{0}(c, \varepsilon)$ be a large enough real number, $P=N^{1 / c}$. We shall prove that the inequality

$$
\left|p^{c}+p_{1}^{c}+p_{2}^{c}+p_{3}^{c}+p_{4}^{c}-N\right|<\varepsilon
$$

is solvable in prime numbers subject to

$$
P / 2<p<2 P, \quad p_{1}, p_{3} \in A, p_{2}, p_{4} \in B .
$$

Set $\Delta=\varepsilon / 2, \delta=\varepsilon / 50, s=20$ and $\Phi(\xi)=\Phi_{20}(\xi)$. From Lemma 2 it follows that the number $J$ of solutions of inequality (5) satisfies

$$
J \geq \frac{1}{\pi} \int_{-\infty}^{\infty} \Phi(\xi) S_{A}^{2}(\xi) S_{B}^{2}(\xi) S(\xi) e^{-i \xi N} d \xi
$$

where

$$
S_{A}(\xi)=\sum_{p \in A} e^{i \xi p^{c}}, \quad S_{B}(\xi)=\sum_{p \in B} e^{i \xi p^{c}}
$$

Therefore

$$
\pi J>J_{1}+J_{2}+J_{3}
$$

where

$$
\begin{aligned}
& J_{1}=\int_{|\xi|<H P^{-c}} \Phi(\xi) S_{A}^{2}(\xi) S_{B}^{2}(\xi) S(\xi) e^{-i \xi N} d \xi \\
& J_{2}=\int_{H P^{-c}<|\xi|<\log P} \Phi(\xi) S_{A}^{2}(\xi) S_{B}^{2}(\xi) S(\xi) e^{-i \xi N} d \xi \\
& J_{3}=\int_{|\xi|>\log P} \Phi(\xi) S_{A}^{2}(\xi) S_{B}^{2}(\xi) S(\xi) e^{-i \xi N} d \xi .
\end{aligned}
$$

From now on, all constants implicit in the Vinogradov symbols $\ll$ and $\gg$ may depend on $c$ and $\varepsilon$.

We estimate $J_{1}$ from below using Lemma 3. If $N_{1}=N-p_{1}^{c}-\ldots-p_{4}^{c}$ then $P^{c} / 2<N_{1}<P^{c}$. Hence

$$
J_{1}=\sum_{p_{1}, p_{3} \in A} \sum_{p_{2}, p_{4} \in B} \int_{|\xi|<H P^{-c}} \Phi(\xi) S(\xi) e^{-i \xi N_{1}} d \xi \gg\left(\frac{P^{c-1}}{\log P}\right)^{2}\left(\frac{P}{\log P}\right)^{2} \frac{P^{1-c}}{\log P}
$$

i.e.

$$
J_{1} \gg \frac{P^{c+1}}{\log ^{5} P}
$$


Now we shall obtain upper bounds for $J_{2}, J_{3}$. Since

$$
\sum_{p_{1}, p_{3} \in A} \sum_{p_{2}, p_{4} \in B} e^{i \xi\left(p_{1}^{c}+p_{2}^{c}-p_{3}^{c}-p_{4}^{c}\right)}
$$

is a non-negative real number for any real $\xi$, Lemma 4 (with $m=10$ ) yields

$$
J_{2} \ll \frac{P}{\log ^{10} P} \sum_{p_{1}, p_{3} \in A} \sum_{p_{2}, p_{4} \in B} \int_{-\infty}^{\infty}|\Phi(\xi)| e^{i \xi\left(p_{1}^{c}+p_{2}^{c}-p_{3}^{c}-p_{4}^{c}\right)} d \xi .
$$

Now we make use of Lemma 1 . For any integer $j$ there exists $t_{j} \in(j-1, j+1)$ such that

$$
J_{2} \ll \frac{P}{\log ^{10} P} P^{c} \log P \sum_{j}\left|\int_{-\infty}^{\infty}\right| \Phi(\xi)\left|e^{i \xi t_{j}} d \xi\right| .
$$

Therefore from the second part of Lemma 2 we derive

$$
J_{2} \ll \frac{P^{c+1}}{\log ^{9} P} \text {. }
$$

For $J_{3}$ we use the trivial estimation of $S(\xi)$ :

$$
\begin{aligned}
J_{3} & \leq P \int_{|\xi|>\log P}\left|\Phi(\xi) S_{A}^{2}(\xi) S_{B}^{2}(\xi)\right| d \xi \\
& \leq \frac{P}{\log ^{10} P} \int_{|\xi|>\log P}\left|\xi^{10} \Phi(\xi) S_{A}^{2}(\xi) S_{B}^{2}(\xi)\right| d \xi
\end{aligned}
$$

i.e.

$$
J_{3} \leq \frac{P}{\log ^{10} P} \sum_{p_{1}, p_{3} \in A} \sum_{p_{2}, p_{4} \in B} \int_{-\infty}^{\infty}\left|\xi^{10} \Phi(\xi)\right| e^{i \xi\left(p_{1}^{c}+p_{2}^{c}-p_{3}^{c}-p_{4}^{c}\right)} d \xi .
$$

Now as in the case of $J_{2}$ we obtain

$$
J_{3} \ll \frac{P^{c+1}}{\log ^{9} P} .
$$

Thus from (6)-(9) we obtain $J \gg P^{c+1} / \log ^{5} P$.

The Theorem is proved.

Acknowledgements. The author would like to thank the referee for several corrections.

\section{References}

[1] Y.-C. Cai, On a diophantine inequality involving prime numbers, Acta Math. Sinica 39 (1996), 733-742 (in Chinese).

[2] M. Z. Garaev, On lower bounds for the $L_{1}$-norm of exponential sums, Math. Notes 68 (2000), 713-720. 
[3] A. Kumchev, A diophantine inequality involving prime powers, Acta Arith. 89 (1999), 311-330.

[4] A. Kumchev and M. B. S. Laporta, On a binary diophantine inequality involving prime powers, in: Number Theory for the Millennium II, A K Peters, 2002, 307-329.

[5] A. Kumchev and T. Nedeva, On an equation with prime numbers, Acta Arith. 83 (1998), 117-126.

[6] I. I. Piatetski-Shapiro, On a variant of Waring-Goldbach's problem, Mat. Sb. 30 (1952), 105-120 (in Russian).

[7] D. I. Tolev, On a diophantine inequality involving prime numbers, Acta Arith. 61 (1992), 289-306.

[8] —, On a system of two diophantine inequalities with prime numbers, ibid. 69 (1995), $387-400$.

Institute of Mathematics

Academia Sinica

Taipei 11529, Taiwan

Republic of China

E-mail: garaev@math.sinica.edu.tw

Received on 13.3.2002

and in revised form on 29.10.2002 